\title{
Evaluación de Control Interno bajo el Método Risicar: Caso servicios bancarios
}

\section{Evaluation of Internal Control under Risicar method: Case banking services}

\author{
Ordoñez-Parra, Janice \\ Universidad Católica de Cuenca \\ Cuenca, Código Postal 010150, Ecuador \\ jordonezp@ucacue.edu.ec
}

\begin{abstract}
Resumen
Pilar fundamental en una institución financiera es el área de servicios bancarios punto clave de contacto que el cliente tiene con la institución, durante los primeros cinco minutos, dependerá del funcionario si gana o pierde a un potencial cliente, de esta manera se considera que todos los procesos que tienden a brindar los diferentes productos y servicios sean de calidad, cumplan con las expectativas y necesidades sin dejar de lado la excelencia en la atención al cliente los cuales están establecidos por las instituciones financieras del país. El mayor interés en el presente trabajo es que sea un aporte y guía de aplicación mediante una eficaz herramienta de evaluación del control interno llamado el método Risicar, con miras a profundizar y mejorar los diferentes procesos dentro de una institución financiera de manera puntal en el área de servicios bancarios con el riesgo potencial de la mala atención, y otros factores amenazantes como el lavado de dinero, el riesgo notable en la banca virtual y canales electrónicos, entre otros. El proceso inicia con la identificación de los riesgos en base al contexto interno y externo esta información ayudará a generar las tablas de los macroprocesos y posteriormente cada uno de los procesos dentro del área de servicios bancarios como son: Apertura de cuentas, chequeras, modificación de datos, cuentas inmovilizadas, banca virtual y canales electrónicos, una vez generada la valoración de los riesgos se realiza la presentación de resultados y correlación del estudio propuesto, para así obtener el mayor riesgo y de igual forma el proceso que genera más riesgo en el área de servicios bancarios. Con esta información se puede mejorar los procedimientos, recomendar las acciones a tomar para responder a las falencias detectadas y dar capacitación a todos los colaboradores del departamento analizado.
\end{abstract}

Palabras clave: Control, Manuales, Risicar, Procesos, Riesgo.

\begin{abstract}
Fundamental prop in a financial institution is the area of bank services key point of contact that the client has with the institution, during the first five minutes, it will depend on the official if it gains or loses a potential client, this way it is considered that all the processes that tend to offer the different products and services should be of quality, should expire with the expectations and needs and there do not leave aside the excellence in the attention to the client which are established by the financial institutions of the country. The biggest interest in the present work is that it is a contribution and application guide by means of an effective tool of evaluation of the internal control called the method Risicar, with a view to deepening and prop improves the different processes inside a way financial institution in the field of bank services with the potential risk of the bad attention, and other threatening factors like the wash of money, the notable risk in the virtual banking and electronic channels, between others. The process initiates with the identification of the risks based on the internal and external context this information it will help to generate the stage of the macro processes and later each of the processes inside the area of bank services as they are: Opening of accounts, checkbooks, modification of information, immobilized accounts, virtual banking and electronic channels, as soon as the evaluation of the risks was generated realizes the presentation of results and interrelation of the proposed study, this way to obtain the biggest risk and in the same way the process that generates more risk in the field of bank services. With this information it is possible to improve the procedures, to recommend the actions to take to answer to the detected bankruptcies and to give training to all the collaborators of the analyzed department.
\end{abstract}

Key words: Control, Manuals, Risicar, Processes, Risk.

\section{Introducción}

Siempre en la vida es importante antes de tomar alguna decisión, tener la seguridad de obtener los mayores beneficios, más aún cuando de por medio está inmerso el riesgo económico que juega el futuro de una persona o empresa. Para ello al momento de trabajar con una 
Institución Bancaria, hay que tener la certeza que ésta tiene que tomar caminos acordes con visión a un mejor futuro. Y de esta forma las partes satisfagan en los mutuos acuerdos apegados a los beneficios que cada uno persigue. Es por esta razón que el entorno económico y todos los elementos que se inmiscuyen en los actos de la economía, repercuten y se vinculan en forma propia a la gestión personal o empresarial, en tal virtud, es necesario tomar en consideración de manera imprescindible las políticas económicas tomadas por el gobierno de turno, las acciones políticas y sociales que acontecen en el ámbito nacional e internacional, así como por algún factor natural que pueda suscitarse, y afectan al normal desarrollo de las actividades propias de los diferentes sectores productivos en el país.

Los nuevos principios elaborados por el Comité de Supervisión Bancaria de Basilea, conocidos como Basilea II, exige que las entidades bancarias incorporen en su actividad la medición de los Riesgos Operacionales. Esto implica una serie de situaciones para ser consideradas hoy en día por las instituciones y en algunos casos ha sido la causa de grandes pérdidas. Gestionar los riesgos es poder identificar en toda una línea de procesos los riesgos que se presentaran, cuál es su magnitud, su ocurrencia, qué efectos acarrean y cómo controlarlos o mitigarlos.

En este contexto, el control interno se lo define como el plan de organización del conjunto de métodos y procedimiento que aseguren que los activos estén debidamente protegidos (...) y que la actividad de la entidad se desarrolle eficazmente según las directrices marcadas por la administración (Estupiñan Gaitan, 2015), y el autor (Mantilla B., 2013) menciona que el informe COSO I establece al control interno "como un proceso, ejecutado por la junta de directores, la administración principal y otro personal de la entidad, diseñado para proveer seguridad razonable en la relación con el logro de los objetivos de la organización”, en los siguientes ambitos o categorías:a. Eficacia y eficiencia de las operaciones, b. Fiabilidad de la información financiera, c. Cumplimeinto de las leyes y reglamentos (Carmona González, 2007).

El punto clave radica en gestionar los problemas ocasionados por distintos segmentos, como sistemas, procesos, recursos humanos, fraudes externos o internos, políticas de manuales a seguir; es decir, el tema operativo de una institución bancaria. En este sentido, según (Mejia, 2006) "Risicar" consiste en una metodología por la cual se aplica un enfoque sistémico para la identificación, medición y monitoreo de riesgos, y posteriormente define las acciones a realizar, con el fin de mitigar los costos ocultos que esos riesgos generan.

El control interno a desarrollar en el área de Servicios Bancarios permitirá evaluar el diseño y funcionamiento de los sistemas establecidos en la Institución para su correcta aplicación, teniendo en consideración algunos aspectos importantes y que contribuirán a precisar las deficiencias administrativas y operativas en los diferentes procesos, incluyendo al análisis de la visión, misión, valores, objetivos y procedimientos en los manuales establecidos.

\section{Estado del arte}

La Ley orgánica de régimen monetario en el art. 24.manifiesta que, el sistema financiero del Ecuador comprende el Banco Central del Ecuador, las instituciones financieras públicas, las instituciones financieras privadas y las demás instituciones controladas por la Superintendencia de Bancos y Seguros.

Según (Comité de Supervisión Bancaria de Basilea, 2006) considera que el riesgo operativo es un factor de gran importancia para los bancos, los cuales deben mantener el capital necesario para protegerse de posibles pérdidas causadas por estos riesgos. El riesgo operativo se define como el riesgo de que se produzcan pérdidas como resultado de procesos, personal o sistemas internos inadecuados o defectuosos, o bien a consecuencia de acontecimientos externos. El riesgo operativo constituye otro ámbito para el que el Comité de Basilea II ha articulado un nuevo método de capital regulador. El mismo que está dispuesto a conceder a los bancos un margen de flexibilidad sin precedentes para que desarrollen un método destinado a calcular el capital necesario para cubrir el riesgo operativo que mejor se ajuste a sus actividades y riesgos subyacentes.

Existen diferentes métodos para establecer la valoración del riesgo, como los descritos por (Mejia, 2006, pág. 80) en su libro "Administración de riegos empresariales":

- Los métodos cualitativos se usan cuando la organización no posee suficiente información sobre la ocurrencia de los riesgos y cuando el costo de obtenerla es mayor que el beneficio. Este análisis utiliza descripciones para mostrar la posibilidad de que los riesgos se presenten (baja, media, alta) y el impacto (leve, grave, catastrófico).

- Los métodos cuantitativos se caracterizan por el uso de modelos matemáticos; entre ellos se cuenta con datos de eventos que tengan una historia conocida, y una frecuencia y variabilidad que permita establecer predicciones.

- En los métodos semi-cuantitativos, a las escalas cualitativas como las mencionadas anteriormente se le asignan valores. Para ello se pueden realizar entrevistas y reuniones de grupos interdisciplinarios. También es posible utilizar diferentes fuentes, como registros históricos, experiencias significativas en el uso de las escalas, prácticas en el sector, literatura publicada, etc.

El método Risicar es una nueva propuesta metodológica surge del estudio de la profesora Rubi Mejia Quijano sobre el tema administración de riesgos, realizado desde 1998 en la Universidad de Medellín y que cobró forma en el proyecto Diseño del modelo de control interno para entidades del Estado, auspiciado por la Agencia de los Estados Unidos para el Desarrollo Internacional, operado por la firma asesora internacional Casals y Associates Inc. Y desarrollado académicamente por la Universidad, sus ventajas se describirán en el transcurso del artículo. 


\section{Metodología}

Para realizar el presente trabajo de evaluación de control interno y el análisis del riesgo operativo y sus resultados como base de aplicación es el método Risicar que es una nueva propuesta metodológica de la profesora Rubí Mejía Quijano sobre el tema de Administración de riesgos y cuyo objetivo es analizar y mejorar los procesos operativos en el área de servicios bancarios.

Algunas ventajas que encontró su autora al utilizar este método son las siguientes:

- Facilidad de aplicación en todo tipo de empresas, tanto pequeñas como grandes, públicas o privadas.

- Su enfoque por procesos, lo que permite administrar los riesgos en forma integral en toda la organización, a través de su modelo de operación.

- La asignación de responsabilidad sobre la administración de riesgos, tanto en el nivel directivo como operativo.

- La creación de cultura de manejo de los riesgos y suministro de herramientas y conocimiento a cada empleado, para asumir su responsabilidad ante ellos.

Iniciaremos con la etapa de identificación de los riesgos con un análisis de contexto (figura 1), para establecer las circunstancias y condiciones en la cual se encuentra la entidad financiera y determinar con mayor precisión los factores que pueden influir en la ocurrencia de los mismos.

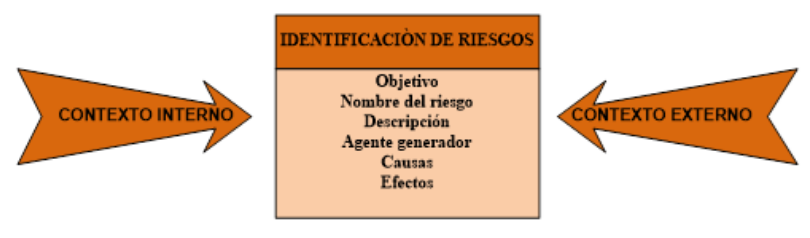

Figura 1. Identificación del riesgo

En lo relacionado al contexto interno se realiza un análisis y conocimiento de la institución desde sus inicios, la misión, visión, principios y valores, organigrama de tal manera que permita tener claramente definido como se lleva a cabo los procesos y el establecimiento de las funciones de las personas que forman parte de la misma, analizando de igual manera las fortalezas, y debilidades que se han presentado en el tiempo. En el contexto externo se analizará la relación que tiene la entidad con los elementos que se encuentran fuera de ella que no son controlables o pueden condicionar el desempeño tanto en aspectos positivos o negativos y que de manera determinante influirían en qué tipo de riesgos pueden propiciarle a la institución.

Dentro de este contexto se considera en primer lugar la relación con el cliente que en la actualidad conoce claramente sobre sus derechos lo que conlleva a que sea mucho más exigente a la hora de acercarse a una entidad financiera para recibir atención sin olvidar también que no tendría razón de existir la entidad, ya que de ellos depende también el logro de los objetivos propuestos.
Otro aspecto a considerar es la relación con el entorno que enmarca la competencia, el estado, el medio ambiente. Una vez determinados estos aspectos se genera el informe de contexto para continuar con la Identificación de riesgos operativos y mecanismos de monitoreo. Para la identificación de riesgos las herramientas a utilizar son: Cuestionarios, observación participante, gráfica de flujos de procesos que manejan actualmente en la entidad e identificar sus riesgos inherentes.

Realizado el análisis interno y externo de la entidad se establecerá el nivel en el cual se realizará la identificación del riesgo y en este caso será en el nivel operativo en cuanto a los procesos que pueden afectar al logro de los objetivos propuestos dentro del área de servicios bancarios presentados en la tabla 1. A continuación se procede a identificar los riesgos en el macroproceso en el área de servicios bancarios. La identificación de riesgos en el área de servicios bancarios es una etapa de vital importancia que permitirá detectar las falencias que se presentan en el trabajo diario, afectando el desarrollo de sus actividades y cumplir con los objetivos propuestos.

Para el trabajo se aplica las tablas que sustentan el desarrollo de la identificación de riesgos en cada uno de los procesos dentro del área de servicios bancarios como son Apertura de cuentas, chequeras, modificación de datos, cuentas inmovilizadas, banca virtual y canales electrónicos, de acuerdo a la aplicación del Método Risicar nueva metodología de la profesora Rubí Mejía Quijano.

Tabla 1

Macroprocesos en el área de servicios bancarios

\begin{tabular}{|c|c|c|c|}
\hline $\begin{array}{l}\text { MACROPROCESO: } \\
\text { OBJETIVO: }\end{array}$ & \multicolumn{3}{|c|}{$\begin{array}{l}\text { Mejorar los tiempos y proces os operativos en los diferentes productos que se brinda a los } \\
\text { clientes en el área de servicios bancarios }\end{array}$} \\
\hline MACROPROCE SO & PROCE SOS & ACT IVIDADES & TAREAS \\
\hline \multirow{15}{*}{$\begin{array}{l}\text { Control Intemo Area } \\
\text { Servicios Bancarios }\end{array}$} & \multirow{2}{*}{ Apertura de Cuentas } & Cuenta corriente & \multirow{2}{*}{$\begin{array}{l}\text { *Cliente solicita apertura } \\
\text { "Ingresar la información en los sistemas para } \\
\text { verificación } \\
\text { *Solicitar documentación } \\
\text { "Realizar apertura cventa corriente o ahorros }\end{array}$} \\
\hline & & Cuenta de ahorros & \\
\hline & & Requerimiento & *Cliente solicita emisión chequera \\
\hline & Chequeras & Reposicion & *Verificacion de datos \\
\hline & & Entrega & 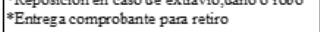 \\
\hline & & Solicitud & iente solicita modificacion \\
\hline & Modificacion de datos & Verificación & $\begin{array}{l}\text { "Ingresar la información en los sistemas para } \\
\text { venificación }\end{array}$ \\
\hline & & Ingreso de datos & $\begin{array}{l}\text { *3olicitar documentación de res paldo } \\
\text { * Realizar modificación en cuenta corriente o }\end{array}$ \\
\hline & & Modificacion & \\
\hline & & Solicitud & ivación \\
\hline & Cuentas inmovilizadzas & Verificación & $\begin{array}{l}\text { inmovilizada } \\
\text { "Ingreas la información en los sistemas para }\end{array}$ \\
\hline & & Ingreso de datos & $\begin{array}{l}\text { ven tacacion } \\
\text { *Solicitar documentación para actualización }\end{array}$ \\
\hline & & Cambio vigencia & *Realizar cambio de vigencia \\
\hline & & Solicitud & $\begin{array}{l}\text { *Cliente solicita activación del servicio } \\
\text { * Solicitar documentación }\end{array}$ \\
\hline & electronicos & $\begin{array}{l}\text { Activación del } \\
\text { servicio }\end{array}$ & $\begin{array}{l}\text { Emitir contrato correspondiente } \\
\text { *Enca virtual de elave para servicio Banca virtual y } \\
\text { Canal electrónico }\end{array}$ \\
\hline
\end{tabular}

A continuación se procede a realizar la identificación de riesgos por cada uno de los procesos que se llevan a 
cabo en el área de servicios bancarios, para el ejemplo lo realizamos con el proceso de apertura de cuentas corrientes, como se puede observar en la tabla 2.

Tabla 2

Proceso Apertura de Cuenta

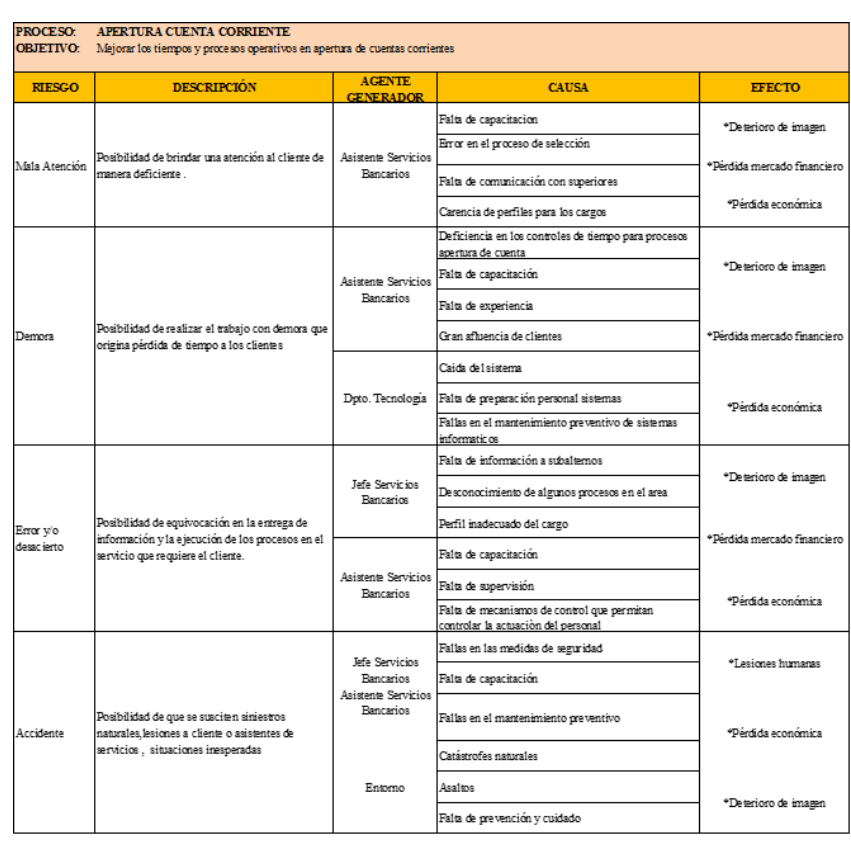

Para continuar se procede a realizar la escala de valoración del riesgo operativo la que aplicaremos una vez realizada la identificación de los riesgos en cada proceso dentro del área de servicios bancarios. Se utilizará las tablas para la calificación de la frecuencia y del impacto sugerido por el método Risicar aplicando cuatro niveles de calificación.

Frecuencia: Esta variable hace referencia al número de veces que un riesgo se puede presentar en un período determinado de tiempo. Mientras exista la posibilidad de que el riesgo se presente con mayor frecuencia, mayor será el valor de su calificación (tabla 3).

Tabla 3

Calificación de frecuencia

\begin{tabular}{|c|l|l|}
\hline \multicolumn{3}{|c|}{ CALIFICACIÓN DE LA FRECUENCIA } \\
\hline VALOR & FRECUENCIA & DESCRIPCIÓN \\
\hline 1 & Baja & Un caso entre 2 y 3 meses \\
\hline 2 & Media & Entre 1 y 5 casos en un mes \\
\hline 3 & Alta & Entre 6 y 10 casos en 15 días \\
\hline 4 & Muy alta & Màs de 10 casos en 8 dias \\
\hline
\end{tabular}

Impacto: La variable impacto se relaciona con las consecuencias que la ocurrencia del riesgo pudiera ocasionarle a la entidad, para la calificación nos basaremos en una tabla que contiene cuatro niveles, similar que en la calificación de la frecuencia, con la variante que los valores crecen en forma geométrica, y se da mayor peso al riesgo que tiene mayor incidencia en el área (tabla 4).

Tabla 4

Calificación del Impacto

\begin{tabular}{|c|l|c|l|l|}
\hline \multicolumn{5}{|c|}{ CALLFICACIÓN DEL IMPACTO } \\
\hline VALOR & IMPACTO & $\begin{array}{c}\text { DESCRIPCIÓN EN } \\
\text { TÉRMINOS } \\
\text { ECONÓMICOS }\end{array}$ & $\begin{array}{l}\text { DESCRIPCIÓN EN } \\
\text { TÉRMINOS } \\
\text { OPERACIONALES }\end{array}$ & $\begin{array}{c}\text { DESCRIPCIÓN EN } \\
\text { TERMINOS DE } \\
\text { IMAGEN }\end{array}$ \\
\hline 5 & LEVE & $\begin{array}{c}\text { Pérdidas hasta } \\
\$ 1000\end{array}$ & $\begin{array}{l}\text { Se interrumpe la } \\
\text { operación menos } \\
\text { de una hora }\end{array}$ & $\begin{array}{l}\text { Solo de conocimiento } \\
\text { en el area de } \\
\text { servicios Bancarios }\end{array}$ \\
\hline 20 & MODERADO & $\begin{array}{c}\text { Pérdidas entre } \\
\$ 1100 \text { y } \$ 5000\end{array}$ & $\begin{array}{l}\text { Se interrumpe la } \\
\text { operación entre dos } \\
\text { y tres horas }\end{array}$ & $\begin{array}{l}\text { Solo de conocimiento } \\
\text { de Jefe inmediato }\end{array}$ \\
\hline 40 & SEVERO & $\begin{array}{c}\text { Pérdidas entre } \\
\$ 5100 \text { y } \$ 10000\end{array}$ & $\begin{array}{l}\text { Se interrumpe la } \\
\text { operación medio } \\
\text { dia }\end{array}$ & $\begin{array}{l}\text { De conocimiento del } \\
\text { cliente interno y } \\
\text { externo }\end{array}$ \\
\hline & & Màs de $\$ 10000$ & $\begin{array}{l}\text { Se interrumpe la } \\
\text { operación } 1 \text { día }\end{array}$ & $\begin{array}{l}\text { De conocimiento } \\
\text { cliente externo y a } \\
\text { nivel local }\end{array}$ \\
\hline
\end{tabular}

En la evaluación se analizan las calificaciones dadas en la etapa anterior y se diseñan los planes de manejo de acuerdo a la prioridad y gravedad de los riesgos. Existirán riegos calificados como inaceptables y severos, los mismos que requieren de una atención inmediata, y en otros casos su calificación será tolerable y aceptable que de igual forma requerirán de acciones a tomar a mediano plazo.

La propuesta del método Risicar es aplicar una matriz de evaluación del riesgo, que está elaborada con las dos variables que componen la calificación del riego (frecuencia e impacto), en las filas se ubican los niveles de calificación de la frecuencia y en las columnas los niveles de calificación de impacto. La multiplicación de estas dos variables determina la zona marcada con las letras:

Tabla 5

Matriz de niveles de calificación

$\mathrm{A}=$ ACEPTABLE
$\mathrm{B}=$ TOLERABLE
$\mathrm{C}=$ GRAVE
$\mathrm{D}=$ INACEPTABLE

De acuerdo a la tabla 5 un riesgo con calificación de cinco y ubicado en la zona marcada con la letra A, no representa peligro para la organización y no requiere de medidas de tratamiento a corto plazo, pues su ocurrencia es baja y su impacto leve. Por el contrario, si un riesgo obtiene una calificación de 160 y se ubicas en la zona marcad con la letra D, la situación de la empresa es inaceptable, porque representa un grave peligro debido a su frecuencia muy alta y su impacto catastrófico; en este caso las medidas de tratamiento de riesgo deben ser inmediatas, porque si se presenta el riesgo, las estabilidad de la empresa puede verse afectada, dado el tamaño y gravedad de la pérdida, como se aprecia en la tabla 6. Para los riesgos ubicados en las otras zonas se toman medidas de forma diferente; por ejemplo, para los riesgos ubicados en la zona $\mathrm{B}$, la respuesta puede 
darse a mediano plazo, con la letra $\mathrm{C}$, la respuesta debe implementarse a corto plazo (Mejia, 2006, pág.96).

Tabla 6

Matriz de evaluación de riesgo: Relación Frecuencia - Impacto

\begin{tabular}{|c|c|c|c|c|c|c|c|c|c|}
\hline \multicolumn{10}{|c|}{ FRE CUE NCIA } \\
\hline 4 & Muy alta & 20 & $\mathrm{C}$ & 40 & $\mathrm{D}$ & 80 & $\mathrm{D}$ & 160 & $\mathrm{D}$ \\
\hline 3 & Alta & 15 & $\mathrm{C}$ & 30 & $\mathrm{D}$ & 60 & D & 120 & $\mathrm{D}$ \\
\hline 2 & Media & 10 & B & 20 & C & 40 & D & 80 & D \\
\hline 1 & Baja & 5 & A & & $\mathrm{C}$ & \multirow{2}{*}{\multicolumn{2}{|c|}{ Ser ero }} & 40 & $\mathrm{D}$ \\
\hline & & \multicolumn{2}{|c|}{ Lere } & \multicolumn{2}{|c|}{ Moderado } & & & \multicolumn{2}{|c|}{ Catastró fico } \\
\hline & & \multicolumn{2}{|c|}{5} & \multicolumn{4}{|c|}{$\frac{\mid}{20}$} & \multicolumn{2}{|c|}{40} \\
\hline
\end{tabular}

\subsection{Presentación de resultados y correlación del estudio propuesto}

Para continuar con la presentación de los resultados y realizado el respectivo análisis de los riesgos por cada proceso objeto de este estudio, en base a la recomendación del método Risicar las calificación se obtiene multiplicando las variables frecuencia e impacto (tabla 7). Adicional se cuenta con la participación del Jefe de Área, y el equipo de trabajo a quienes también se les solicita su colaboración con encuestas y entrevista personal, información que será de gran utilidad para definir las medidas de tratamiento de los riesgos.

Tabla 7

Calificación: Relación frecuencia Impacto

\begin{tabular}{|c|c|c|c|c|c|}
\hline PROCE SO: & APE RT URA CUE & CORRIENIE & & & \\
\hline RIE SGO & FRECUENCIA & IMPACTO & CALIFICACIÓN & EVALUACIÓN & $\begin{array}{c}\text { MEDIDA DE } \\
\text { TRAT AMIE NTO }\end{array}$ \\
\hline \begin{tabular}{|l|} 
Mala Atención \\
\end{tabular} & 2 & 20 & 40 & Inaceptable & Prevenir, Proteger, Transfereri \\
\hline Demora & 3 & 20 & 60 & Inaceptable & Prevenir, Proteger, Transfenir \\
\hline Error y o desacierto & 2 & 10 & 20 & Grave & Prevenir, Proteger, Transfenir \\
\hline \begin{tabular}{|l|} 
Accidente \\
\end{tabular} & 1 & 10 & 10 & Tolerable & Prevenir, Proteger \\
\hline
\end{tabular}

Luego de realizar la identificación de los riesgos, se procede a dar a cada uno el tratamiento que corresponda de acuerdo a la evaluación obtenida. Y para ello se utilizará la matriz de respuesta ante los riesgos que recomienda la

Tabla 8

Matriz de la frecuencia y el impacto

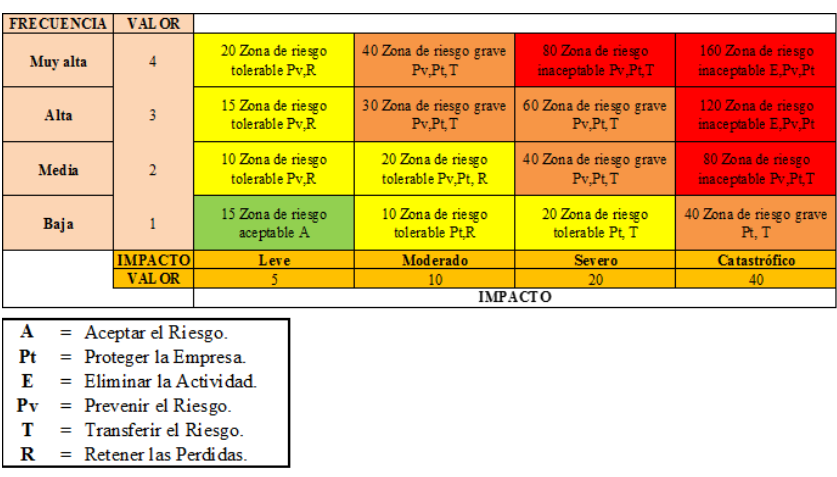

aplicación del método con el cual estamos trabajando (tabla 8).

A continuación se ponderan los riesgos y procesos identificados en las etapas anteriores según el nivel de peligrosidad que representa, de acuerdo al criterio del Jefe de servicios bancarios. Estableciendo un porcentaje a cada riesgo cuya distribución equivale al $100 \%$ (tabla 9).

Tabla 9

Resultados ponderación de riesgos y procesos

\begin{tabular}{|c|c|}
\hline \multicolumn{2}{|c|}{ PONDE RACIÓN DE RIESGOS } \\
\hline MALA ATENCIÓN & $45 \%$ \\
\hline DEMORA & $25 \%$ \\
\hline ERROR Y/O DESACIERTO & $20 \%$ \\
\hline ACCIDENTE & $10 \%$ \\
\hline TOTAL & $100 \%$ \\
\hline
\end{tabular}

\begin{tabular}{|c|c|}
\hline \multicolumn{2}{|c|}{ PONDE RACIÓN DE LOS PROCE SOS } \\
\hline APERTURA DE CUENTA CORRIENTE & $30 \%$ \\
\hline APERTURA CUENTA DE AHORROS & $30 \%$ \\
\hline CHEQUERAS & $10 \%$ \\
\hline MODIFICACION DE DATOS & $5 \%$ \\
\hline CUENTAS INMOV ILIZADAS & $15 \%$ \\
\hline CANALES ELEC TR ONICOS, BANCA VIR TUAL & $10 \%$ \\
\hline TOTAL & $100 \%$ \\
\hline
\end{tabular}

\subsection{Matriz de priorización de riesgos y tareas}

Con la aplicación de la matriz de priorización de riesgos y tareas (tabla 10) se establecerá de acuerdo al resultado obtenido, el mayor riesgo y de igual forma el proceso que genera más riesgo en el área de servicios bancarios. Con esta información se puede mejorar los procedimientos y dar la capacitación correspondiente a todos los responsables y colaboradores del departamento analizado.

Tabla 10

Matriz de priorización de riesgos y tareas

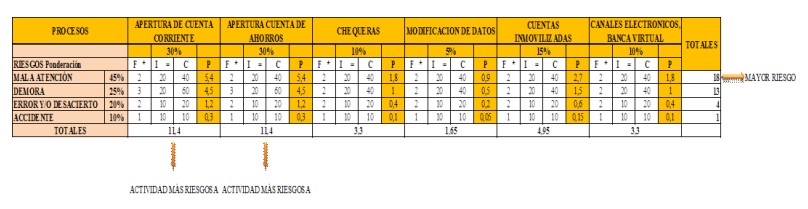

De acuerdo a la información obtenida en la matriz de priorización de riesgos se puede establecer los mayores riesgos y actividades vulnerables, permitiendo al Jefe inmediato del área de servicios bancarios definir los controles necesarios y recomendar las mejoras en los manuales de procedimientos existentes para una efectiva administración de riesgos.

En cuanto al tema de riesgos y que requiere una aplicación de manera urgente en primer lugar es la mala atención (tabla 11) que sin lugar a dudas afecta notablemente en el 
Tabla 11

Matriz de priorización de riesgos

\begin{tabular}{|c|l|}
\hline PRIORIDAD & \multicolumn{1}{|c|}{ RIE SGOS } \\
\hline 1 & MALA ATENCION \\
\hline 2 & DEMORA \\
\hline 3 & ERROR Y/O DESACIERTO \\
\hline 4 & ACCIDENTE \\
\hline PRIORIDAD & \multicolumn{1}{|c|}{ PROCE SOS } \\
\hline $\mathbf{1}$ & APERTURA DE CUENTA CORRIENTE \\
\cline { 2 - 3 } & APERTURA CUENTA DE AHORROS \\
\hline 2 & CUENTAS INMOVII IZADAS \\
\hline \multirow{2}{*}{3} & CHEQUERAS \\
\cline { 2 - 2 } & CANALES ELECTRONICOS, BANCA VIR TUAL \\
\hline \multirow{2}{*}{4} & MODIFICACION DE DATOS \\
\hline
\end{tabular}

cumplimiento de los objetivos que tiene la entidad. Seguido por los riegos en cuanto a demora y error y/o desacierto por ello el objetivo central es buscar mejoras en los manuales de procedimientos para lograr resultados positivos y que permitan disminuir y eliminar las falencias presentadas en la actualidad.

\subsection{Acciones a tomar para responder a las fallas y debi- lidades presentadas}

En el área de servicios bancarios de manera general cuentan con un manual de procedimientos para cada uno de los procesos objeto de este estudio, todo el personal del departamento debe realizar su trabajo en base a las normativas expuestas, las cuales deben ser analizadas y reformadas para obtener mejores resultados.

A continuación se presenta las tablas de medidas de tratamiento y controles propuestos para ser aplicados en el área de servicios bancarios. En base a esta información se procederá con el plan y acciones inmediatas a tomar en los procesos que hemos analizado.

Estos resultados serán expuestos a la Alta Dirección, Jefes de área y responsables de la elaboración de los manuales de procedimientos para que se garanticen mejoras en los mismos, estableciendo una fecha límite para se lleve a cabo. Es de vital importancia el contar con un grupo de personas que se responsabilicen por la ejecución del mismo con toda la información que se presenta en este trabajo.

Al tener como resultado que el riesgo potencial y que afecta notablemente en el área es la mala atención, seguida del riesgo demora factores que indudablemente impiden el cumplimiento de los objetivos de la institución. Se deberá tomar medidas que permitan ejercer control sobre su efectividad, si bien es cierto existe manuales, se debe realizar un actualización y capacitar nuevamente a todo el personal que forma parte del área involucrada.

Además considerar la posibilidad de crear un sistema que permita realizar evaluaciones mensuales a las asistentes de servicios bancarios y sus Jefes inmediatos, con lo cual se detectan las falencias presentadas y se diseña un plan de acción inmediato para lograr corregir las debilidades, mantener las fortalezas y redireccionar las acciones, de tal manera que se mejore los procesos en conjunto dentro del área objeto de este estudio.

Para el efecto se presenta los resultados que permite considerar de mejor manera los controles actuales y los propuestos en el proceso de apertura de cuenta corriente, y se cierra el proceso con la elaboración del flujograma que ayudará a identificar las actividades fundamentales en el área de servicios bancarios.

Riesgo Mala Atención.

Calificación 40.

Evaluación Inaceptable.

Medida de tratamiento Prevenir, proteger, transferir.

Riesgo Demora.

Calificación 60 .

Evaluación Inaceptable.

Medida de tratamiento Prevenir, proteger, transferir.

Riesgo Error y/o desacierto.

Calificación 20.

Evaluación Grave.

Medida de tratamiento Prevenir, proteger, transferir.

Riesgo Accidente.

Calificación 10.

Evaluación Tolerable.

Medida de tratamiento Prevenir, proteger.

Control Actual :

Establecer los lineamientos que deben seguir en la ejecución de las actividades que se realizan para atender de manera ágil y eficiente los requerimientos para apertura de cuenta corriente.

Se realiza una evaluación semestral, la misma que efectúa el jefe inmediato

Recientemente se implementó el manual de salud y seguridad ocupacional.

\section{Control Propuesto :}

Actualización de manuales con la colaboración del personal que labora en las áreas involucradas en lo referente a cuenta corriente.

Capacitación al personal del área de servicios bancarios para refrescar procedimientos y liberar las dudas e inquietudes en el trabajo diario.

Realizar un control y evaluación mensual a las asistentes de servicios bancarios para detectar falencias y buscar mejora inmediata.

\section{Resultados}

Una vez realizado el proceso de evaluación de control interno en el área de servicios bancarios el cual género como resultado que el nivel de riesgo más alto es la mala atención que afecta al desarrollo de las diferentes actividades al igual que el factor demora que indudablemente impiden el cumplimiento de los objetivos de la institución. Como resultados se llevó a cabo la socialización del mismo a los directivos y el personal involucrado para aplicar las medidas de tratamiento Prevenir, proteger, transferir 


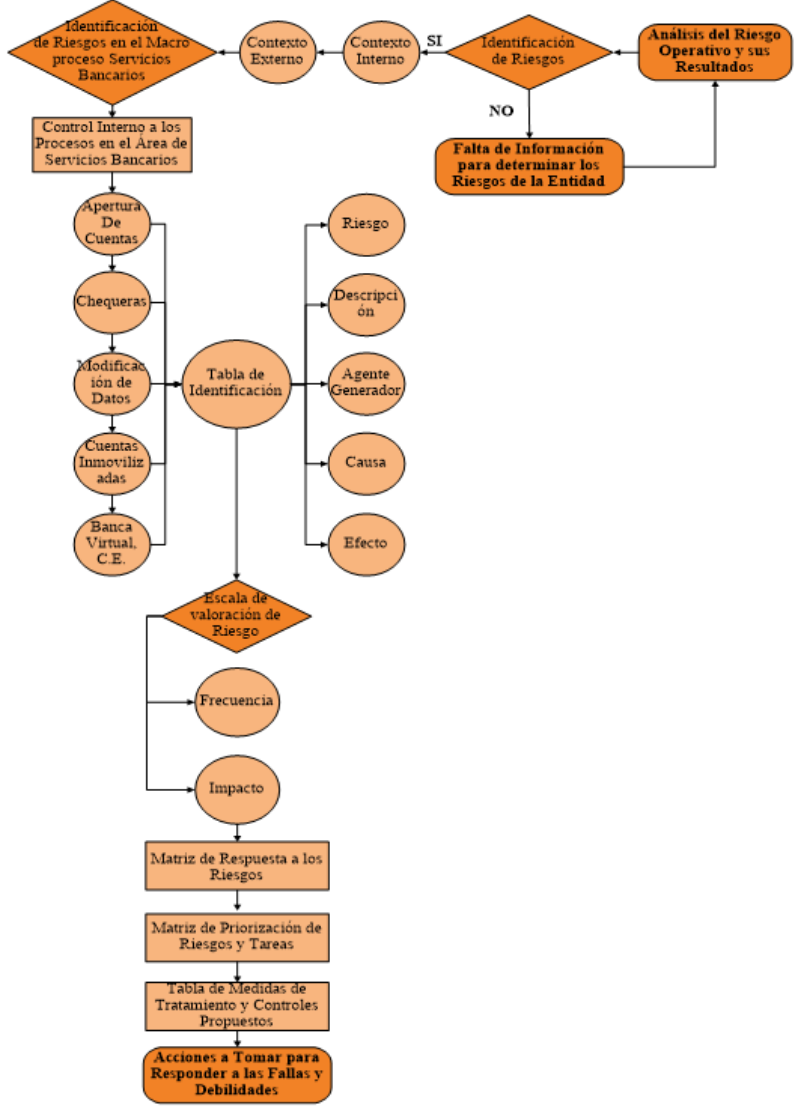

Figura 2. Flujograma de procesos en el área de servicios bancarios

y en primera instancia se procedió con la capacitación del personal que labora en el área de servicios bancarios, motivar a que realicen su trabajo con empeño, conocer y actualizarse en todos los procedimientos establecidos, de igual manera se explicó que se llevará un mejor control de procedimientos y tiempos mediante la aplicación de un sistema que permite realizar evaluaciones mensuales a las asistentes de servicios bancarios y sus Jefes inmediatos de esta manera se detecta las falencias presentadas y se aplica un plan de acción inmediato para logar corregir las debilidades, mantener las fortalezas y redireccionar las acciones.

De igual manera se generó cambios en los procesos que se llevan a cabo en las diferentes actividades como apertura de cuentas, chequeras, modificación de datos, cuentas inmovilizadas, banca virtual y canales electrónicos los cuales se plasmaron en los flujogramas de procesos (figura 2) lo cual facilita el desenvolvimiento y fluidez en las operaciones y el personal tiene mucho más claro las funciones y actividades a desarrollar.

Otro punto fue el tema de los manuales de procedimientos en lo referente a las aperturas de cuentas, activaciones, modificaciones de datos se realizó una actualización y se consideró algunos aspectos para unificar e impartir a sus colaboradores y trabajen de manera conjunta al momento de solicitar los documentos correspondientes al cliente, sobre todo evitarle las molestias que ocasionaban en la actualidad especialmente el factor demora.

Actualmente están generando los procesos de una manera más ágil de igual manera facilitan a los clientes el poder acceder a los diferentes productos y servicios en menos tiempo y la calidad, calidez y buen trato al cliente está haciendo la diferencia en la institución.

\section{Conclusiones}

El presente trabajo tiene como finalidad el lograr mejoras en los procedimientos que se realizan en un departamento de vital importancia dentro de la institución financiera como es servicios bancarios, ya que al ofrecer un buen servicio puede hacer la diferencia entre captar un cliente pasajero o un cliente fiel a la Institución considerando también que hoy en día la calidad del servicio no solo implica un producto que se ofrece, involucra otros ámbitos como el buen trato, sistemas y procesos.

Partiendo de este aspecto se realizó el análisis de los diferentes procedimientos que se llevan a cabo en esta área aplicando el método Risicar que es una nueva propuesta implementada por la profesora Rubí Mejía, del cual se obtuvo como resultado un riesgo potencial denominado mala atención que afecta notablemente al desarrollo de las actividades cotidianas de la institución, y el factor demora que sin lugar a duda impiden el cumplimiento de los objetivos.

Otro punto a considerar es el tema de los manuales de procedimientos en lo referente a las aperturas de cuentas, activaciones, modificaciones de datos se debe realizar una actualización y considerar algunos aspectos para unificarlos e impartirlos a sus colaboradores y trabajen de conjunta al momento de solicitar los documentos correspondientes al cliente, sobre todo evitarle las molestias que ocasionan en la actualidad.

En cuanto al tema del servicio banca virtual y canales electrónicos son medios prácticos que han ido creciendo a pasos agigantados y con la tendencia a tener su banco en todas partes, en la entidad se cuenta con un sistema actualizado y constantemente se realizan innovaciones para precautelar la seguridad y confidencialidad de la información de sus clientes.

Otro aspecto importante es el disminuir notablemente el riesgo al cliente al no traer consigo dinero en efectivo mediante las diferentes opciones que se brinda desde la comodidad de la casa u oficina y de igual manera al encontrarse en cualquier establecimiento dentro o fuera del país. Ayudando al control de gastos porque puede disponer del dinero depositado en sus cuentas las 24 horas los 365 días del año.

En la actualidad todas la instituciones financieras están amenazadas de ser víctimas por parte de el crimen organizado para el lavado de dinero y cada vez adquieren más fuerza , provocados en gran medida por una creciente complejidad 
en los negocios, el impacto de la virtualidad, principalmente en las transferencias de fondos entre diferentes países, los riesgos implícitos en el trato con diferentes personas y culturas que tienen un mayor uso instrumental de las nuevas tecnologías que al parecer son reales pero están en el anonimato en gestión de negocios generando mayor riesgo y exigen que las entidades cuenten con controles eficientes y eficaces que les permita mitigar estos riesgos.

De ahí la importancia que todos los empleados deben estar actualizados en todos los procesos que implican el tema de apertura de cuentas corrientes, ahorros y servicio de banca virtual sin dejar de lado en ningún momento la ética que nos muestra y permite saber en conciencia lo que se puede y lo que no se debe hacer ante posibles situaciones que se presentan en el trabajo diario para proceder con las notificaciones al oficial de cumplimiento responsable para que se aplique la justicia y el resguardo de los bienes de sus clientes y de la institución.

\section{Recomendaciones}

Se recomienda en base a la información presentada, exponerla ante la alta dirección y responsables de los procesos para que se garantice una actualización y mejoras en los manuales existentes estableciendo una fecha límite para que se lleve a cabo. Y realizar una capacitación a todo el personal que forma parte del área de servicios bancarios. Se debe tener claro que todos los departamentos dentro de una entidad bancaria son importantes, ya que al fallar uno de ellos disminuye la calidad en el servicio que percibe el cliente, aunque los demás estén desarrollando su trabajo de manera correcta.

Considerar la posibilidad de crear un programa que permita realizar evaluaciones mensuales al personal que labora en las áreas involucradas, de esta manera detectar las falencias presentadas y tener un plan de acción para tomar los correctivos necesarios con la finalidad de cumplir con las expectativas y necesidades de los clientes. A su vez el sistema permitirá verificar el cumplimiento de los estándares dentro de la entidad.

Involucrar a las asistentes de servicios bancarios para que contribuyan con ideas a sus Jefes inmediatos para mejorar el trabajo diario, estén conscientes y reconozcan los ciclos de servicio que involucra a cada cliente, en esa medida podrá colaborar obteniendo una visión completa de lo que su cliente necesita y espera.

Si bien es cierto en la entidad se cuenta con excelentes mecanismos para brindar seguridad y confiabilidad a sus clientes en el uso de la banca virtual y canales electrónicos sería conveniente que a más de la información que se entrega al cliente en el momento de la activación de dichos servicios se haga llegar informativos mensuales mediante los mismos canales y folletos a sus direcciones de correspondencia de tal forma que estén actualizados de las diferentes maneras que pueden operar los delincuentes y así evitar ser víctimas de algún tipo de estafa.
En lo referente a la prevención y control del lavado de dinero en cuentas corrientes, cuentas de ahorros y Banca virtual se puede realizar capacitaciones semestrales por cuanto la delincuencia organizada va perfeccionando sus métodos en la parte operativa y es importante el reforzar los conocimientos ante diferentes indicios, actividades inusuales o sospechosas de lavado de dinero y activos. Teniendo siempre presente que la regla de oro es "conocer el cliente y su mercado" al igual que "conocer a su empleado". Al combinar una excelente capacitación y aplicación de las 5 "C" seguro se obtendrá aún mejores resultados de los que ya se tienen en la Institución.

\section{Referencias}

Carmona González, Y., M; Barrios Hernández. (2007). Nuevo paradigma del control interno y su impacto en la gestión pública. Economía y Desarrollo, 141, 152-171. Descargado de Recuperadodehttp: / /www.redalyc.org/ articulo.oa?id=425541595009

Comité de Supervisión Bancaria de Basilea. (2006, jun). Convergencia internacional de medidas y normas de capital. Descargado de http: / /www.bis.org/ publ/bcbs128_es.pdf

Estupiñan Gaitan, R. (2015). Control interno y fraudes: Análisis del informe coso $i$, ii y iii con base en ciclos transaccionales (3ra. Edición ed.). ECOE Ediciones.

Mantilla B., S. A. (2013). Auditoría de control interno (Era. Edición ed.). ECOE Ediciones.

Mejia, R. (2006). Administración de riesgos un enfoque empresarial (1era. ed.). Medellín, Colombia,: Fondo Editorial Universidad EAFIT.

Recibido: 13 de julio de 2016

Aceptado: 23 de agosto de 2017

Ordoñez-Parra, Janice: Docente de la Unidad Académica de Administración de la Universidad Católica de Cuenca, Licenciada en Administración de Empresas, Contadora Público, Ingeniera Comercial, Magister en Contabilidad y Finanzas con Mención en Gerencia y Planeamiento Tributario. 